\title{
A Parametric Discriminative Approach for Skin color Detection by Training Weak Learners on Normalized Chrominance and Luminance
}

\author{
Faisal Jamal Nasir \\ University of Engineering and \\ Technology \\ Peshawar, Pakistan
}

\author{
Nasir Ahmad \\ University of Engineering and \\ Technology \\ Peshawar, Pakistan
}

\author{
Syed Shadab Ali Shah \\ University of Engineering and \\ Technology \\ Peshawar, Pakistan
}

\begin{abstract}
This paper presents a novel approach for the detection of skin color in image or video, captured through ordinary web camera. The TSL color space is used due to its specialty in distinguishing among the skin and Non-skin color. To label the skin colors, a classifier based on adaboost algorithm has been trained. To validate the performance of the classifier, a database of skin colors was developed using different color tones ranging from fair to deep.
\end{abstract}

\section{General Terms}

Skin color detection, TSL color space, Adaboost color classifier, Skin color tones mixing.

\section{Keywords}

TSL, HCI, Adaboost, RGB2TSL.

\section{INTRODUCTION}

Skin color detection is of great importance in various human computer interaction (HCI) applications due to its common use as a pre-processing step in human body parts detection and tracking. Due to the advances in robotics technology, human gestures recognition has got great attention of the researchers which also can be assisted by skin color detection. Color detection is also of much importance in development of a lot of mobile camera software to enhance face colors so that to adorn a picture. It is also used as indication of human presence in a picture or video frame.

The two approaches are typically used for skin detection are texture based approach and skin color tone based approach. There are three main problems usually faced in the skin color detection approach [1]. Firstly, selection of the color space that can perform best for the skin detection, secondly, the modeling of skin color space, and thirdly, the separation of objects from background. This paper deals with the first two of these problems. Skin color distribution can be modeled primarily by many ways such as histogram models [2], single/Gaussian mixture models [3], and table look-up methods [4]. Many of these techniques are not effective when the illumination conditions vary rapidly and for the whole range of skin tones from fair to deep color tone. To deal with these problems a few skin detection approaches use color constancy or dynamic adaptation method. In color constancy approaches, the images are first color corrected to normalize illumination. The skin-color modeling and detection are then applied on these preprocessed images. In dynamic adaptation techniques, the existing skin-color model is transformed to the changing illumination conditions [5].
In this paper the skin color labeling has been improved by the application of skin oriented color scheme like TSL and by training adaboost classifier on the basis of color perception. The approach is based on the fact that skin colors vary more in intensity than in luminance [6], and that TSL T and $S$ component in the TSL space capture most of the color information. Therefore, in this work, only Tint and Saturation are used for training the classifier which gives superior performance compared to previously reported results.

\section{RELATED WORK}

Skin detection techniques based on pixel tone have longer history than that of region based methods. Kruppa et al. [7] proposed a region based skin color detection technique and tested it on the famous Compaq dataset. He used color and spatial distance to extract skin region from the image and named it as superpixel. Results were further purified using conditional random field (CRF). Despite of its high calculation cost, technique achieved superb results with $91.17 \%$ true positive rate and $13.12 \%$ false negative rate.

Yang and Ahuja [8] tried to estimate a probability density function of human skin color by finite Gaussian mixture model. They performed many experiments to show that the estimated Gaussian mixture model fits skin images from a large database.

There are many types of color spaces which can be used for modeling of skin color. Sebe et al. [9] proposed that the skin-color clusters in normalized RGB space have relatively less variance than in RGB color space and hence have shown to be good for skin-color modeling and detection. The Commission Internationale de l'Eclairage (SCIE) system introduced CIE-XYZ color space as an effort to correspond to the color matching characteristics of human visual system. Calculation cost lies in conversion of RGB to CIE$\mathrm{XYZ}$ yet it has been used by Chen and Chiang [10] due to its perceptual uniformity and device independence.

Perceptual color spaces like HSV that can be deduced by Non Linear mapping of RGB have been used by Zhu et al. [11]. HSV color space defines colors as Hue - the property of color that changes during transformation in red to green, Saturation - the property that changes in transformation from red to orange and value - the intensity or lightness of color. Being invariant to white and ambient light and surface orientation of light it has proven a very good choice in skin color detection.

Hsu et al. [12] checked the performance of orthogonal color spaces like YCbCr, YIQ, YUV, YES to reduce the redundancy present in RGB color channels. He tried to show that 
these spaces are a favorable choice for skin detection by separating the luminance and chrominance components. After the selection of suitable color space for skin color detection, the next stage is of clustering of color pixels into skin and non skin color. Higher is the control on illumination component, better will be the clustering of colors [13].

Skin color detection may also be considered as binary classification problem. Dai et al. [14] used fixed range values for thresh holding between skin and non skin color pixels. He defined a range of values in components of different color spaces as skin color and rest as non skin, quantized colors into histogram bins to differentiate a large number of objects and then converted them into probability distribution. It was demonstrated that due to a reasonable difference in skin and non skin classes a fast and accurate classifier can be built to detect skin in unconstrained environment like web cam. Histogram based Bayes classifier also known as skin probability map (SPM) has also been used for skin color detection [15] due to its high computation speed (only needs two table lookups) and simplicity.

Researchers also have contributed by using skin distribution models like Single Gaussian Model (SGM) and Gaussian Mixture Model (GMM). SGM tries to cluster different skin color tones under controlled environment on the basis of an ROC (Region of Convergence) curve calculated from training data [16]. Effort was made to model skin color more effectively and accurately by clustering a wider range of skin color tones. Increasing the number of parameters in GMM improves modeling on the cost of higher computation. Terrillon et al. [17] presented many statistical tests and proved that GMM is more robust representation of skin color that can accommodate large variations in color space, highlights and shadows depending on type of color space chosen. Artificial Neural Network (ANN) based techniques includes multilayer perception (MLP) by Phung et al. [18], Fuzzy Logic (FL) combined with Probabilistic and Neural Network (PNN) by Anagnostopoulos et al. [19]. MLP is the most prominent neural networks for its non-linear input output relation but its performance is dependent on many factors like number of hidden layers, number of hidden nodes and learning rate. Classification obtained from neural network can be enhanced more by applying Gaussian. Anyhow selection of good non-skin samples is a challenge. SOM provides an unsupervised competitive method for clustering of high dimensioned data for generalization.

A good skin detector is the one that can detect a large verity of skin colors e.g. light, Asian, yellow, deep and Tan under different illumination conditions and with complex background. The major problem with most of the existing techniques is that only a specific skin tone range or specific illumination conditions have been focused [20]. The performance of skin classifiers may vary depends on choice and size of test dataset and time taken in training and classification.

\section{DESCRIPTION OF PROPOSED METHOD}

\subsection{Color space selection}

TSL color space has been used for skin color coding. It is a perceptual color space. Tint is the hue with added white. It is the way to differentiate between colors stimuli being red, green, blue or white. Saturation is colorfulness of a stimuli and Lightness shows that how much a color is bright. TSL normalizes Luminance and Chrominance.
Colors provided by web Cam used to be in RGB format which is then converted into TSL by using equation $3.1,3.2$ and 3.3.

$$
\begin{gathered}
T=\left\{\begin{array}{l}
\frac{1}{2 \pi} \arctan \frac{r^{\prime}}{g^{\prime}}+\frac{1}{4} \quad \text { if } g^{\prime}>0 \\
\frac{1}{2 \pi} \arctan \frac{r^{\prime}}{g^{\prime}}+\frac{3}{4} \quad \text { if } g^{\prime}<0 \\
0 \quad \text { if } g^{\prime}=0
\end{array}\right. \\
S=\sqrt{\frac{9}{5}\left(r^{\prime 2}+g^{\prime 2}\right)} \\
L=0.299 R+0.587 G+0.114 B
\end{gathered}
$$

Where

$$
r=\frac{R}{R+G+B} \quad g=\frac{G}{R+G+B} \quad r^{\prime}=r-\frac{1}{3} \quad g^{\prime}=g-\frac{1}{3}
$$

Above equations show that in transformation from RGB to TSL space colors have been normalized hence reduced in sensitivity against chrominance variations. The advantage of TSL color space lies in better clustering of skin colored pixels on TS space as shown in the Fig.1

For skin color detection adaboost classifier has been trained on only $\mathrm{T}$ and $\mathrm{S}$ values of TSL color space. adaboost is an adaptive boosted learning machine that attempts to create a strong classifier from a number of weak classifiers, formulated by "YoavFreund and Robert Schaphire" [21]. In adaboost first a model is built for training data. This model is then corrected by removing errors. Similarly model is modified again and again until the training set is perfectly predicted.

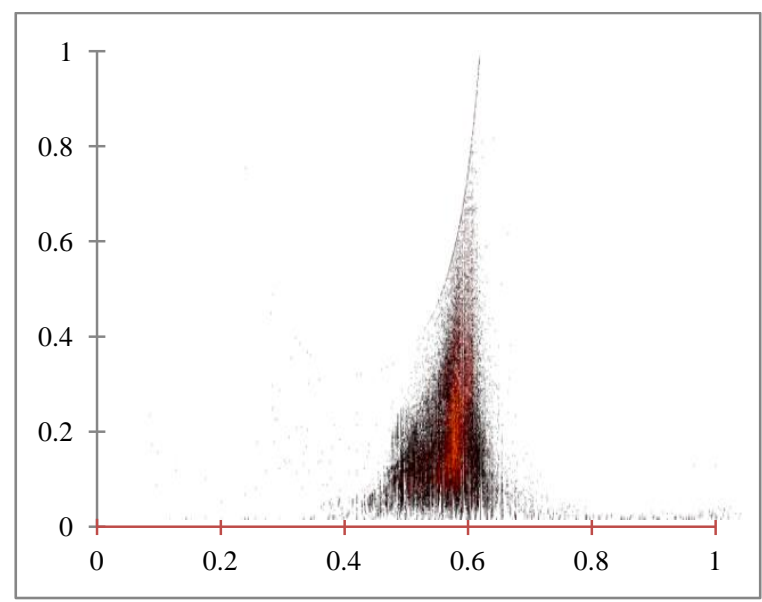

Fig 1: TS heat plot of skin data. Red glow shows more no. of pixel concentration

\subsection{Adaboost classifier}

An adaboost classifier is a weighted combination of classifiers. Boosting trains a model by sequentially training a new simple model based on the errors of previous model. We start on by learning a very simple predictor and then evaluate its errors and focus the next predictor by getting these examples right. This procedure tends to discover the data points hard to predict and then focus later classifier on predicting these examples better. As a result of combining many of these week learners we make a very strong complex classifier. 
A flowchart describing the adaboost algorithm is given in Fig. 2.

The methodology of training a classifier by boosting is given below.

Let we have training data set $\left(\mathrm{x}_{1}, \mathrm{y}_{1}\right) \ldots . .\left(\mathrm{x}_{\mathrm{n}}, \mathrm{y}_{\mathrm{n}}\right)$,

Where $x i \in X$ is an instance of the data to be classified, and yi $\in\{-1,1\}$ is the result of binary classification.

First we initialize weights as given.

$W l(i)=D(i), D(i)=\frac{1}{(2 m)}$ or $\frac{1}{(2 l)}$,

Where $\mathrm{m}, 1$ are number of positive and negative samples respectively.

For each classifier ht, $(\mathrm{t}=1 \ldots . \mathrm{T})$

Normalize weights $q t(i)=\frac{W t(i)}{\sum_{j=1}^{n} W t(j)}$

A classifier $\mathrm{h}$ is trained for each feature $\mathrm{f}$ and the one with lowest error $\mathcal{E}$ is selected.

Weights are updated as given.

$W(t+1)(i)=W t(i) \beta e i$

Where:

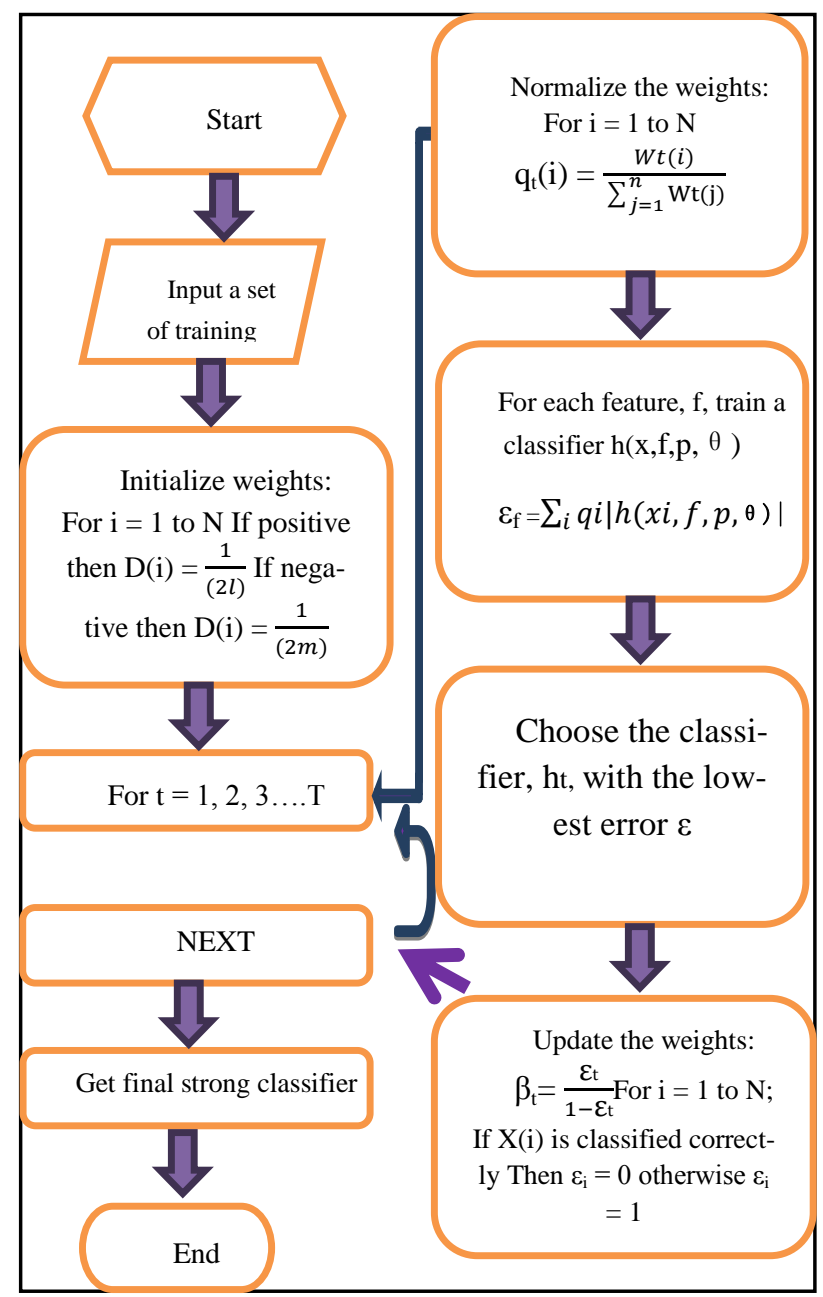

Fig 2: flowchart of adaboost algorithm $\mathrm{e}_{\mathrm{i}}=1$ (if sample is correctly classified otherwise -1 )

$\beta=\frac{\varepsilon \mathrm{t}}{1-\varepsilon \mathrm{t}}$

The final classifier so obtained is given as:

$h(x)=\left\{\begin{array}{ccl}1 & \text { if } & \sum_{t=1}^{\mathrm{T}} \underset{\mathrm{t}}{\alpha} \operatorname{tht}(\mathrm{x})>\frac{1}{2} \sum_{\mathrm{t}=1}^{\mathrm{T}} \underset{\mathrm{a}}{\alpha} \\ 0 & \text { otherwise }\end{array}\right.$

Where: $\alpha \mathrm{t}=\frac{1}{\beta \mathrm{t}}$

Fig: 3 shows how step by step application of week learner's result in a strong classifier. + and - signs represent two type of classes. After application of each classifier, weights are changed in order to remove the error of the current classification in the next one.

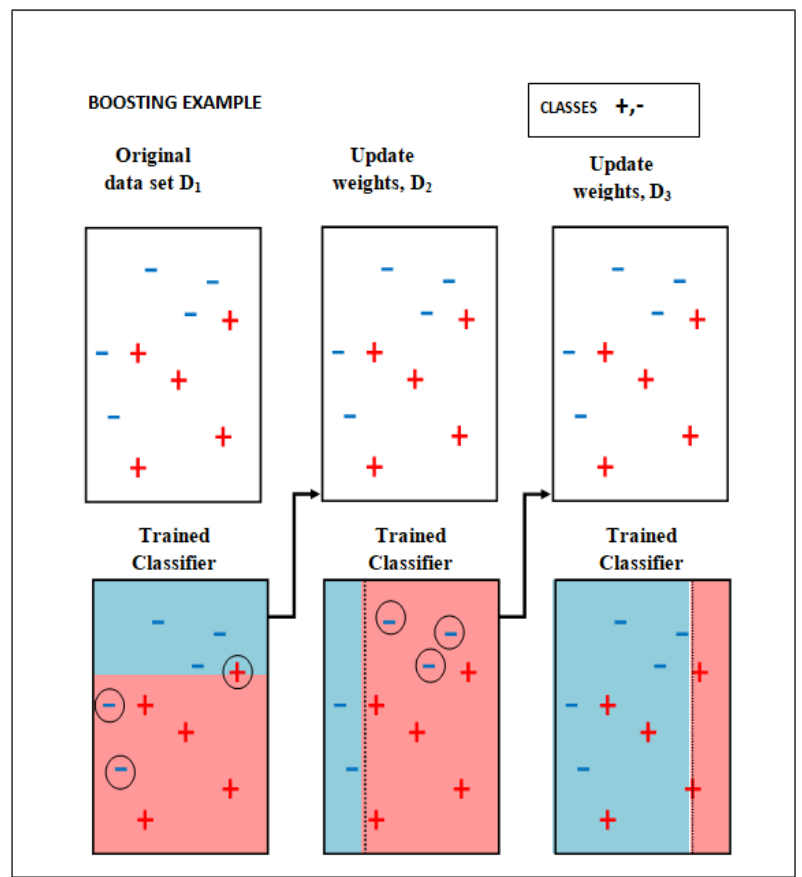

Fig 3: Example of adaboost, step by step application of week classifiers

\subsection{TSL skin color database}

A fine collection of skin patches of various tones is collected by using matlab imfreehand function and the respective RGB values are stored in a text file. These RGB values are fed to RGB2TSL function in order to get TSL values. Similarly there have also been chosen many negative samples and converted to TSL. As shown in the Fig 4. 


\begin{tabular}{|lccc||}
\hline $\mathbf{T}$ & $\mathbf{S}$ & $\mathbf{L}$ & class \\
0.514938 & 0.1461840 & 0.575 & 1 \\
0.514938 & 0.1461840 & 0.575 & 1 \\
0.514938 & 0.1472332 & 0.571 & 1 \\
0.514938 & 0.1472332 & 0.571 & 1 \\
0.514938 & 0.1472332 & 0.571 & 1 \\
0.514938 & 0.1482975 & 0.568 & 1 \\
0.510243 & 0.1504783 & 0.565 & 1 \\
0.514938 & 0.1504731 & 0.560 & 1 \\
0.510243 & 0.1527076 & 0.557 & 1 \\
0.073702 & 0.0722022 & 0.730 & -1 \\
0.073702 & 0.0722022 & 0.730 & -1 \\
0.073702 & 0.0722022 & 0.730 & -1 \\
0.073702 & 0.0722022 & 0.730 & -1 \\
0.073702 & 0.0722022 & 0.730 & -1 \\
0.073702 & 0.0722022 & 0.730 & -1 \\
0.073702 & 0.0722022 & 0.730 & -1 \\
0.073702 & 0.0722022 & 0.730 & -1 \\
0.073702 & 0.0722022 & 0.730 & -1 \\
0.073702 & 0.0722022 & 0.730 & -1 \\
0.073702 & 0.0722022 & 0.730 & -1 \\
& & & \\
\hline
\end{tabular}

Fig 4: TSL values for skin \& non skin colors respectively

$\mathrm{T}$ and $\mathrm{S}$ values so collected are used to train adaboost classifier and results are then checked. The proportion of different color tones in the skin color database have been changed many times so that to get a most appropriate classifier working well for all type of skin color tones. It have been noticed that by keeping fair tone ration $50 \%$ to $55 \%$ more than other color tones and deep color tone only 5 to 10 percent of other tones produced the best results. So the final database of TSL colors having the above mentioned ratio of tones was reserved for adaboost training and future work.

\section{EXPERIMENTAL RESULTS}

We tested our skin detector for classification of different skin color tones separately and found the results shown in Table1.

Table 1: Results of adaboost skin detector on different color tones of VOC2010 dataset

\begin{tabular}{|c|c|c|c|}
\hline Type of skin & No of pixels & $\begin{array}{c}\text { True } \\
\text { Positives } \\
(\%)\end{array}$ & $\begin{array}{c}\text { False } \\
\text { Negatives } \\
(\%)\end{array}$ \\
\hline
\end{tabular}

\begin{tabular}{|l|l|l|l|}
\hline Fair & 184489 & 92.0933 & 7.9067 \\
\hline Light & 347859 & 97.1937 & 2.8063 \\
\hline Tan & 120135 & 97.0467 & 2.9533 \\
\hline Medium & 445221 & 97.8020 & 2.1980 \\
\hline Deep & 162592 & 99.6218 & 0.3782 \\
\hline
\end{tabular}

It is evident that the classifier shows best results even on the deep color tone. We also tested our classifier on phung skin dataset and the results found are given in Table 2.

Table 2: Results of adaboost skin detector on Phung dataset

\begin{tabular}{|c|c|c|c|c|}
\hline $\begin{array}{c}\text { No of } \\
\text { pixels } \\
\text { tested }\end{array}$ & $\begin{array}{c}\text { True } \\
\text { positive }\end{array}$ & $\begin{array}{c}\text { True } \\
\text { negative }\end{array}$ & $\begin{array}{c}\text { False } \\
\text { positive }\end{array}$ & $\begin{array}{c}\text { False } \\
\text { negative }\end{array}$ \\
\hline 443402960 & 99.20 & 82.3433 & 17.6567 & 0.8004 \\
\hline
\end{tabular}

Fig 5 shows the visual results of application of adaboost skin detector on Phung dataset [22].

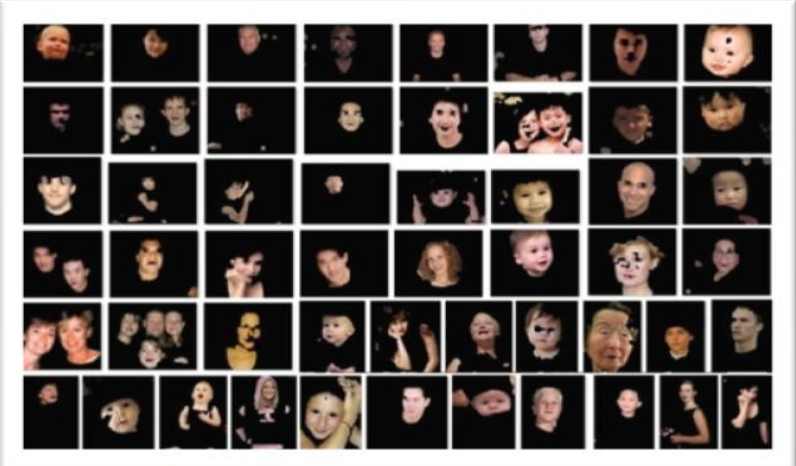

Fig 5: Application of Skin detection algorithms on Phung skin dataset

TS color space has also been compared to other color spaces for different color tones and results are shown in the plot of Fig 6 to Fig 9. 

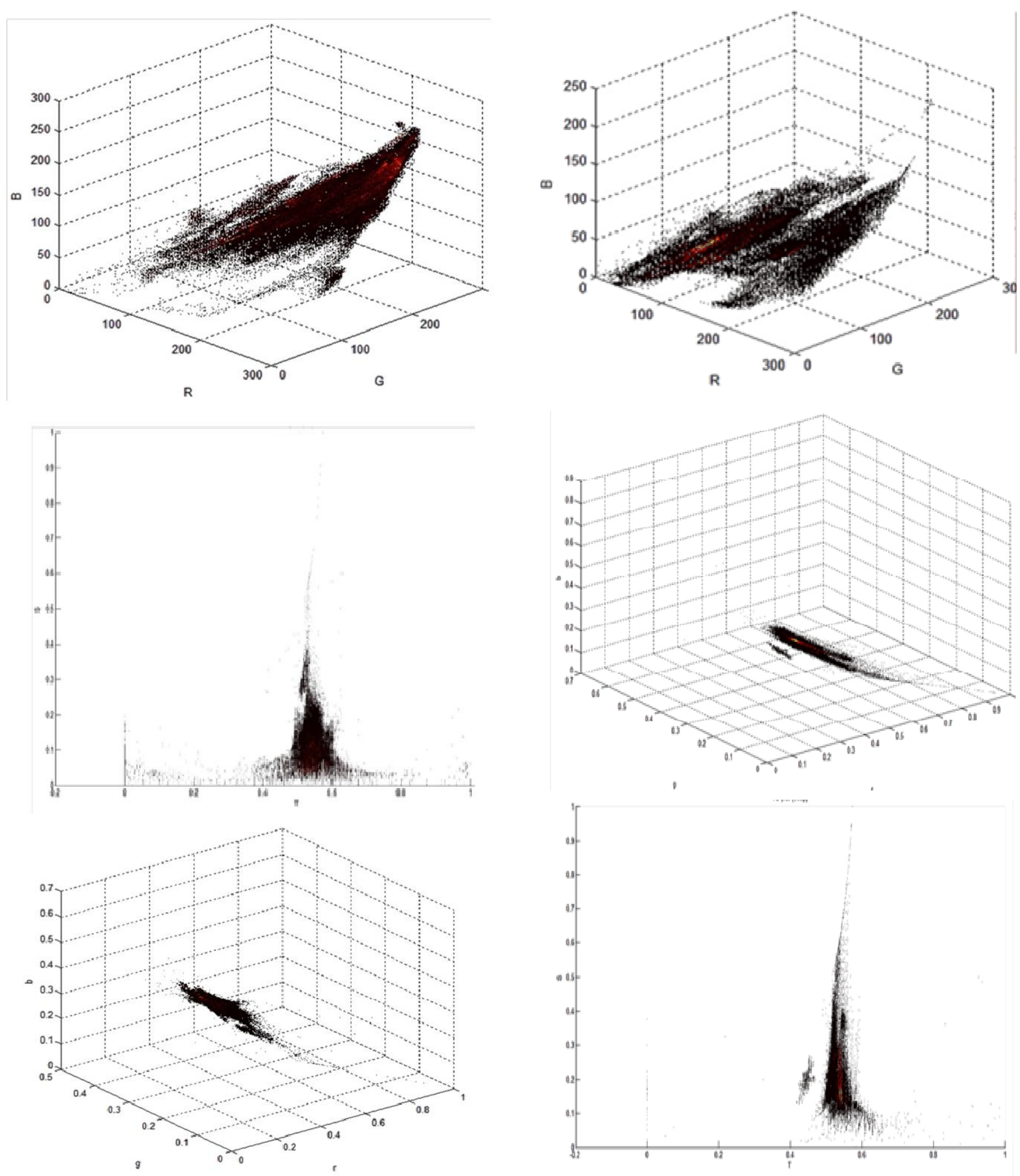

Fig 6: Plot of fair skin color using RGB (top), TS (middle) and Normalized color spaces (bottom) respectively

Fig 7: Plot of deep skin color using RGB (top), TS (middle) and Normalized (bottom) color spaces 

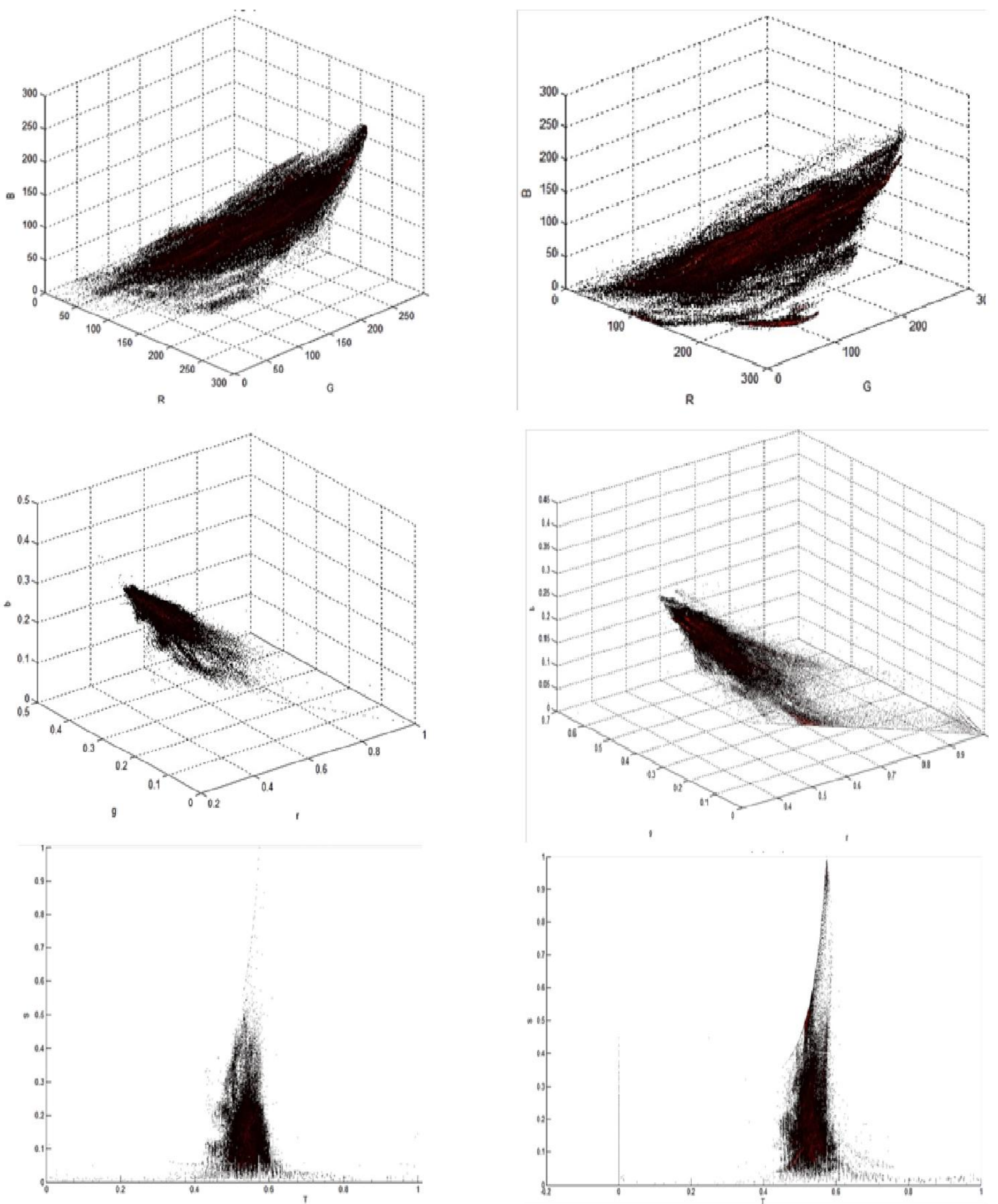

Fig 8: Plot of light skin color using RGB, TS and Normalized color spaces respectively

Fig 9: Plots of medium skin color using RGB, Normalized and TS color space respectively 


\section{SUMMARY}

A huge database of skin and non skin color pixels were prepared from a large variety of pixels of skin colors in appropriate proportion and an adaboost classifier was trained on these pixels in order to produce best detection results. TSL color space was used to store pixel data as it is a skin oriented color space distinguishing the pixels on the basis of skin and non skin color basis. When plotted against different color tones, it was observed that despite of other color spaces there was a very little variation in the cluster position of TSL color space proving its feasibility for detection of a large variety of skin color tones.

\section{REFERENCES}

[1] Vezhnevets, V., Sazonov, V. and Andreeva, A., 2003, September. A survey on pixel-based skin color detection techniques. In Proc. Graphicon (Vol. 3, pp. 8592).

[2] Khan, R., Hanbury, A., Stöttinger, J. and Bais, A., 2012. Color based skin classification. Pattern Recognition Letters, 33(2), pp.157-163.

[3] Yang, M.H. and Ahuja, N., 1999, January. Gaussian mixture model for human skin color and its applications in image and video databases. In Storage and Retrieval for Image and Video Databases (SPIE) (pp. 458-466).

[4] Gourier, N., Hall, D. and Crowley, J.L., 2004, August. Estimating face orientation from robust detection of salient facial structures. In FG Net Workshop on Visual Observation of Deictic Gestures (Vol. 6).

[5] Sun, H.M., 2010. Skin detection for single images using dynamic skin color modeling. Pattern recognition, 43(4), pp.1413-1420.

[6] Yang, J., Lu, W. and Waibel, A.1998, "Skin-color modeling and adaptation", In Asian Conf. on Computer Vision, pp. 687-694. Springer Berlin Heidelberg.

[7] Kruppa, H., Bauer, M.A. and Schiele, B. 2002, "Skin patch detection in real-world images", In Joint Pattern Recognition Symposium pp. 109-116, Springer Berlin Heidelberg.

[8] Yang, M.H. and Ahuja, N.1998, "Gaussian mixture model for human skin color and its applications in image and video databases", In Electronic Imaging'99 Int. Society for Optics and Photonics, pp. 458-466.

[9] Sebe, N., Cohen, I., Huang, T.S. and Gevers, T., 2004, "Skin detection: A bayesian network approach", In Pattern Recognition, ICPR 2004. Proc of the 17th Int. Conf. on vol. 2, pp. 903-906
[10] Chen, C. and Chiang, S.P., 1997. "Detection of human faces in colour images", In IEE Proc-Vision, Image and Signal Processing, 144(6), pp.384-388.

[11] Zhu, Q., Cheng, K.T., Wu, C.T. and Wu, Y.L., 2004, "Adaptive learning of an accurate skin-color model", In Automatic Face and Gesture Recognition, Proc. Sixth IEEE Int. Conf. on pp. 37-42.

[12] Hsu, R.L., Abdel-Mottaleb, M. and Jain, A.K., 2002, "Face detection in color images" In IEEE Trans. on pattern analysis and machine intelligence, 24(5), pp.696-706.

[13] Yang, J., Lu, W. and Waibel, A., 1998, "Skin-color modeling and adaptation", In Asian Conf. on Computer Vision pp. 687-694. Springer Berlin Heidelberg.

[14] Dai, Y. and Nakano, Y., 1996, "Face-texture model based on SGLD and its application in face detection in a color scene", Pattern recognition, 29(6), pp.10071017.

[15] Fleck, M., Forsyth, D. and Bregler, C., 1996. "Finding naked people", In Computer Vision-ECCV'96, pp.593-602.

[16] Kakumanu, P., Makrogiannis, S. and Bourbakis, N., 2007, "A survey of skin-color modeling and detection methods" In Pattern recognition, 40(3), pp.1106-1122.

[17] Terrillon, J.C., Shirazi, M.N., Fukamachi, H. and Akamatsu, S., 2000, "Comparative performance of different skin chrominance models and chrominance spaces for the automatic detection of human faces in color images". In Automatic Face and Gesture Recognition, Proc. Fourth IEEE Int. Conf. on pp. 54-61.

[18] Phung, S.L., Chai, D. and Bouzerdoum, A., 2001, “A universal and robust human skin color model using neural networks", In Neural Networks, Proc. IJCNN'01. Int. Joint Conf. on vol. 4, pp. 2844-2849.

[19] Anagnostopoulos, I., Anagnostopoulos, C., Loumos, V. and Kayafas, E., 2003, "A probabilistic neural network for human face identification based on fuzzy logic chromatic rules" In IEEE MEDO3.

[20] Kakumanu, P., Makrogiannis, S. and Bourbakis, N., 2007, "A survey of skin-color modeling and detection methods" In Pattern recognition, 40(3), pp.1106-1122.

[21] Freund, Y. and Schapire, R.E., 1995, "A desiciontheoretic generalization of on-line learning and an application to boosting", In European Conf. on computational learning theory pp. 23-37.

[22] Phung, S.L., Bouzerdoum, A. and Chai, D., 2005, "Skin segmentation using color pixel classification: analysis and comparison" In IEEE Trans. on pattern analysis and machine intelligence, 27(1), pp.148-154. 